Revue d'histoire de l'Amérique française

REYUE D.HISTOIRE DE L'AMÉRIQUE FRANÇAISE

COSANDEY, Roland, André GAUDREAULT et Tom GUNNING, dir., Une invention du diable? Cinéma des premiers temps et religion/An Invention of the Devil? Religion and Early Cinema. Sainte-Foy et Lausanne, Presses de l'Université Laval et Éditions Payot, 1992. 383 p. 48 \$

\title{
Yves Lever
}

Volume 47, numéro 1, été 1993

URI : https://id.erudit.org/iderudit/305192ar

DOI : https://doi.org/10.7202/305192ar

Aller au sommaire du numéro

\section{Éditeur(s)}

Institut d'histoire de l'Amérique française

\section{ISSN}

0035-2357 (imprimé)

1492-1383 (numérique)

Découvrir la revue

Citer ce compte rendu

Lever, Y. (1993). Compte rendu de [COSANDEY, Roland, André GAUDREAULT et

Tom GUNNING, dir., Une invention du diable? Cinéma des premiers temps et religion/An Invention of the Devil? Religion and Early Cinema. Sainte-Foy et Lausanne, Presses de l'Université Laval et Éditions Payot, 1992. 383 p. 48 \$]. Revue d'histoire de l'Amérique française, 47(1), 108-110.

https://doi.org/10.7202/305192ar d'utilisation que vous pouvez consulter en ligne. 
COSANDEY, Roland, André GAUDREAULT et Tom GUNNING, dir., Une invention du diable? Cinéma des premiers temps et religion/An Invention of the Devil? Religion and Early Cinema. Sainte-Foy et Lausanne, Presses de 1'Université Laval et Éditions Payot, 1992. 383 p. 48\$

Domitor, l'Association internationale pour le développement de la recherche sur le cinéma des premiers temps, tenait son premier colloque international à Québec en juin 1990. L'expression «des premiers temps» signifie ici de la naissance du cinéma (1895) jusqu'à environ 1914. André Gaudreault, de l'Université Laval (maintenant à l'Université de Montréal), était le responsable du colloque, et cet ouvrage bilingue en rassemble les communications.

Dans le programme de la rencontre, le titre Une invention du diable se lisait sans point d'interrogation. C'était refléter surtout la conception qu'on en a eue en contexte québécois où le cinéma en soi, indépendamment des films projetés, a été combattu pendant trente ans parce que considéré comme «corrupteur», «école du soir tenue par le diable», «dénationalisateur», «antireligieux», «délétère», «source de tous les vices», etc. La connaissance d'une partie de la situation internationale a permis de nuancer l'expression et de lui conférer un caractère humoristique. Elle n'en reflète pas moins la vision 
trouble que les religions chrétiennes ont entretenue sur le cinéma jusqu'à la Seconde Guerre mondiale.

Le cinéma des premiers temps est, comme le rappelle Jacques Malthête (p. 227), «un objet d'étude diabolique»; les catalogues, qui ne décrivent pas toujours ce que l'on voit sur l'écran, nous manquent souvent et les bandes peuvent être, comme on l'a vu, à versions multiples. Et puis la copie sauvegardée est-elle conforme à un hypothétique original? Est-elle complète? A-t-elle été remontée? Si c'est le cas, à quelle occasion? dans quel but? pour satisfaire quel public ou quel exploitant? Comment a-t-elle été acquise? et par quelles mains est-elle passée? Comment se présentent les autres copies nitrates du même film, si elles existent... et nous n'avons plus les yeux de ceux pour qui ces bandes ont été conçues!» Cela explique pourquoi les actes de ce genre de colloque, plus que ceux des autres sciences historiques, présentent un aspect un peu agaçant de «recherche en cours» (work in progress), d'inachevé, de partiel. On aurait pu souhaiter que pour la publication, un des chercheurs consacre un substantiel chapitre à une synthèse de ce que le colloque a permis de dégager, non seulement à travers les communications, mais aussi avec les échanges qui les suivaient. Celui de Janet Staiger, «Conclusions and New Beginnings», n'est pas la synthèse à laquelle le lecteur aurait pu s'attendre; elle a toutefois le mérite de relancer l'étude du sujet dans d'autres voies, notamment celle de la représentation des femmes ou celle des réalisations par des femmes.

Une première partie regroupe les communications portant sur «le cinéma vu par les Églises», non seulement les films à sujet religieux, mais l'ensemble des projections. On y constate que là où on utilisait déjà des photographies ou des diapositives pour la catéchèse (Italie, France, Belgique, etc.), les séances de cinéma sont considérées comme un auxiliaire encore plus efficace, selon le précepte de saint Grégoire le Grand voulant que «L'homme a besoin d'être pris par les sens; les images sont les livres de ceux qui n'en ont point d'autres». Ailleurs (Espagne, Québec, Russie, etc.), après un accueil enthousiaste des premières Passions (surtout parce qu'elles se présentent sous le mode documentaire - reportage sur celle d'Oberammergau par exemple alors qu'elles en sont le plus souvent des reconstitutions), les Églises opposent un refus assez catégorique à l'ensemble du phénomène cinématographique. Le fait est constaté, mais trop peu expliqué, me semble-t-il. Je regrette aussi qu'il manque à cet éventail des textes sur les religions autres que le christianisme (judaïsme, islamisme, spiritualités orientales) ou sur certains pays, le Canada par exemple, dont on ne sait à peu près rien sous ce rapport. La partie la plus générale intéressera tous les historiens, les trois autres s'adressant surtout aux spécialistes du cinéma.

Ce refus des Églises se manifeste surtout après 1905, quand le langage et l'esthétique deviennent plus complexes, quand la fiction se développe aux dépends du documentaire. C'est l'objet de la deuxième partie qui examine surtout les «questions de narration» reliées à la Passion filmée et aux films hagiographiques. De toutes les parties, la plus courte est sans doute la plus innovatrice tout en étant la plus inachevée; elle pose davantage de questions théoriques qu'elle n'apporte de réponses, mais on y constate que le film dit 
religieux est un des meilleurs lieux pour développer l'ensemble de la théorie du cinéma, y compris dans ses relations avec le public.

Les troisième et quatrième parties, qui n'en font qu'une en réalité, apportent des «études de cas» dans la représentation du spirituel et du religieux. On y considère certains films en particulier, des courants ou des thèmes spécifiques (l'utilisation du crucifix comme accessoire, métaphysique de l'apparition, la spiritualité de Griffith telle que révélée par le montage, etc.). Tout cela sera fort utile à l'historien qui tentera une synthèse sur le cinéma des premiers temps. Je regrette toutefois que presque personne ne semble intéressé à creuser ce qui se dégage de ces cas au plan de l'imaginaire.

Un index des titres de films, des noms de personnes et d'institutions cités complète l'ouvrage. Il n'en sera que plus utile comme instrument de recherche. L'ajout d'une bibliographie aurait été apprécié. 